\title{
Embryogenèse somatique et organogenèse in vitro chez la luzerne : évaluation des potentiali- tés de divers génotypes
}

\author{
Sylvie BIANCHI, Pascal FLAMENT $\left({ }^{*}\right) \&$ Yvette DATTÉE $\left({ }^{* *}\right)$ \\ A.D.A.R. \\ (*) Boursier M.R.E.S. \\ (**) I.N.R.A., Laboratoire d'Amélioration des Plantes, U.A. 115, Université Paris XI Bâtiment 360, F 91405 \\ Orsay Cedex
}

RÉSUMÉ

\begin{abstract}
Vingt individus issus de populations ou cultivars tétraplö̈des et diploïdes de Medicago sativa L. et de l'espèce Medicago falcata L. ont été évalués pour leur aptitude à la régénération à partir de cals. Les différences interet intra-populations ou cultivars sont très importantes. Les cals issus de 6 génotypes ont permis la régénération de plantes soit par organogenèse ( 2 génotypes) soit par embryogenèse somatique (4 génotypes).

La qualité et le rendement des embryons somatiques ont été améliorés par l'utilisation d'une nouvelle séquence de milieux (UM-UMo).

Deux génotypes de $M$. sativa tétraplö̈des, l'un issu de la population chinoise «Mestnaya », l'autre du cultivar "Europe » ont un potentiel embryogène et de régénération très élevé, nettement supérieur à Regen $\mathrm{S}$ utilisé comme témoin.
\end{abstract}

Mots clés additionnels : Medicago, culture de tissus

Additional key words : Medicago, tissue culture.

\section{INTRODUCTION}

La luzerne (Medicago sativa L.) est, parmi les légumineuses, celle qui a été la plus étudiée en culture in vitro. Dès 1964, CLEMENT réussit à régénérer des plantes à partir de cals. En 1972, SAUNDERS \& BINGHAM mettent au point une séquence de 2 milieux permettant la néoformation de bourgeons et l'obtention de plantes. Puis Bingham et al. (1975) obtiennent après 2 cycles de sélection récurrente, une population (Regen S) ayant un taux élevé de plantes aptes à la régénération $(67 \mathrm{p} .100)$. Par la suite, divers auteurs ont décrit l'embryogenèse somatique de plusieurs cultivars de M. sativa (LuPotTo, 1983, NOVAK \&
KONECNA, 1982). Il est aussi possible de régénérer des plantes de luzerne à partir de suspensions cellulaires (Mc COY \& BINGHAM, 1977) et de protoplastes (KAO \& MiChAYluK, 1980 ; Dos SANTOS et al., 1980). Des hybrides somatiques entre $M$. sativa et Medicago falcata L. ont été obtenus par TEOULE (1983).

Le fait le plus marquant rapporté par tous les auteurs est la grande différence d'aptitude à la régénération intra-populations et inter-populations. MITTEN et al. (1984) et BROWN \& ATANASSOV (1985) ont observé une variabilité génotypique importante parmi un grand nombre de populations américaines et canadiennes testées pour leur aptitude à régénérer in vitro. La luzerne $M$. sativa, autotétraploïde et allogame, est hétérozygote et, de ce fait, présente une forte hétéro- 
généité génétique inter- et intra-population (ou cultivar).

L'objectif est ici d'évaluer l'aptitude à la régénération de plusieurs génotypes toujours individualisés, provenant de populations ou cultivars tétraploïdes et diploïdes de $M$. sativa et de l'espèce $M$. falcata.

Cette évaluation a pour but d'obtenir un système efficace de régénération et de trouver, notamment parmi les cultivars intéressants du point de vue agronomique, les génotypes les plus performants en culture in vitro.

Elle a eu pour préliminaire le choix d'un témoin sélectionné dans la population Regen S. L'aptitude à la régénération de 18 génotypes a alors été testée, par rapport à ce témoin, sur la séquence de milieux défi nie par BINGHAM et al. (1975).

Puis, une autre séquence de milieux a été utilisée et les potentiels embryogènes des 3 meilleurs génotypes ont été comparés.

\section{MATÉRIEL ET MÉTHODES}

Les diverses espèces ou origines sont présentées au tableau 1. Les plantes utilisées ont été cultivées en serre à partir, soit de graines, soit de boutures. Pendant les jours courts la photo-période est maintenue artificiellement à $16 \mathrm{~h}$, par éclairage d'appoint.

Les pétioles sont prélevés sur les dernières et avantdernières feuilles dépliées avant l'apex, stérilisés à l'hypochlorite de calcium à 7 p. 100 pendant $10 \mathrm{mn}$ et rincés 2 fois à l'eau stérile. Ils sont alors coupés en morceaux d'environ $2 \mathrm{~mm}$ et mis en culture dans des boîtes de Pétri contenant le milieu de culture gélosé. Les cultures sont maintenues à $28^{\circ} \mathrm{C}$, avec une photopériode de $16 \mathrm{~h}$ et une intensité lumineuse d'environ $14 \mathrm{~W} / \mathrm{m}^{2}$.

Les 2 premières séries d'expériences utilisent la séquence décrite par SAUNDERS \& BinghaM (1972) qui comprend un premier milieu d'induction des cals (BII) contenant $2 \mathrm{mg} \cdot 1^{-1}$ de $2,4-\mathrm{D}$ et $2 \mathrm{mg} \cdot \mathrm{1}^{-1}$ de kinétine puis un milieu de régénération (BOI2Y) sans régulateurs de croissance. Par la suite, les cals sont repiqués sur le milieu $\mathrm{BOI} 2 \mathrm{Y}$ tous les mois.

La séquence comprenant le milieu décrit pour le tabac par UCHIMIYA \& MURASHIGE (1974) avec $2 \mathrm{mg} . \mathrm{l}^{-1}$ de $2,4 \mathrm{D}$ et $0,25 \mathrm{mg} . \mathrm{l}^{-1}$ de kinétine (UM) suivi du même milieu sans régulateurs de croissance (UMo) a été utilisée par la suite.

Pour les comparaisons du nombre d'embryons sur la séquence de milieux UM-UMo, seuls les cals de

TABLEAU I

Espèces et provenances utilisées pour les expérimentations.

Species, cultivars and populations used for the experiments.

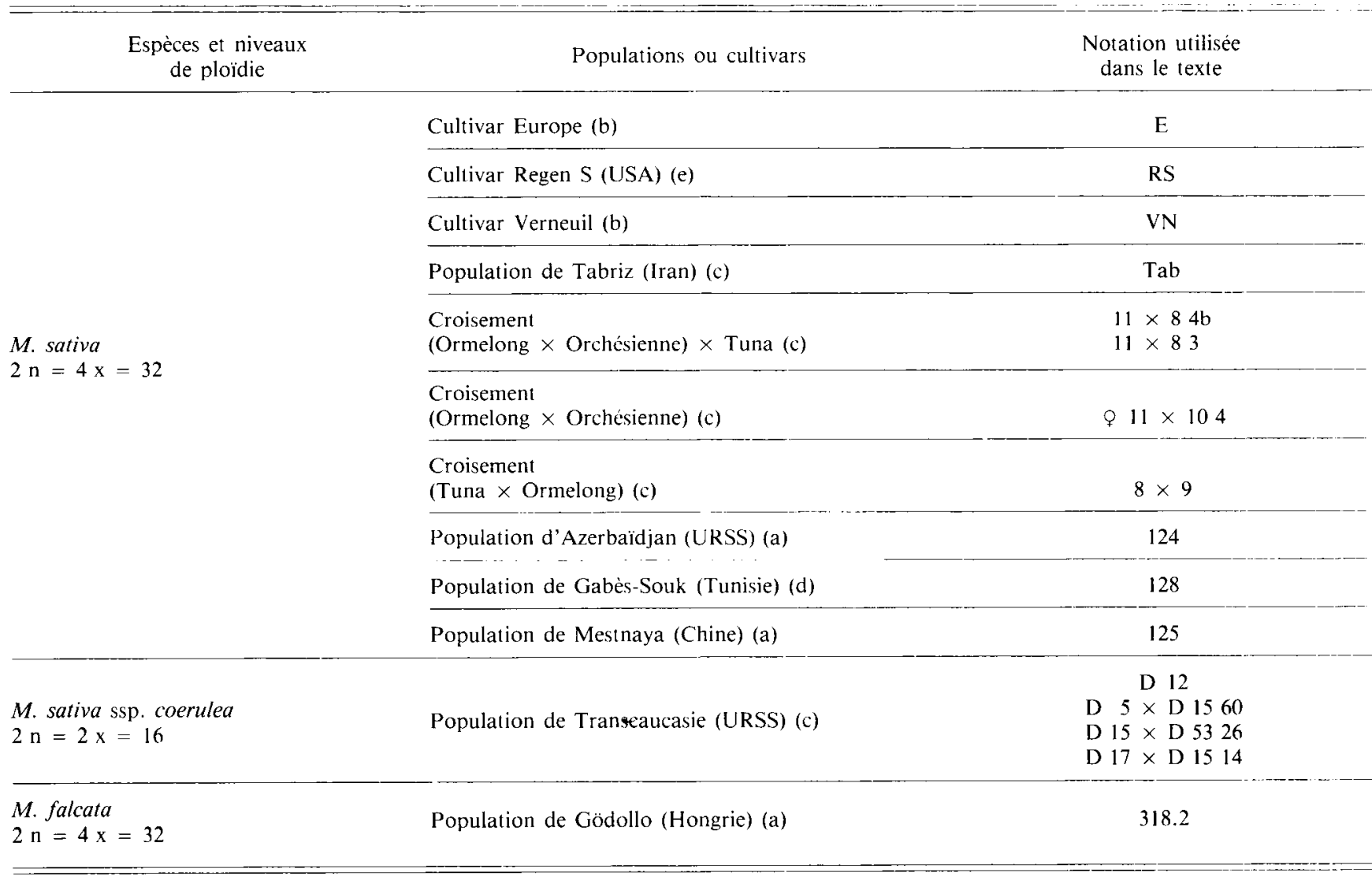


même taille (environ $10 \mathrm{~mm}$ ) sont repiqués après 1 mois.

Le nombre d'embryons par cal est déterminé sous la loupe binoculaire entre 2 semaines et 2 mois après le repiquage sur le milieu UMo.

Les embryons, détachés des cals, se développent et donnent des plantules qui sont repiquées en terre dans une serre. Pour les observations cytologiques les cals sont fixés dans l'alcool acétique (3/1), déshydratés par une série de bains dans l'alcool et le xylène, puis inclus dans la paraffine. Les coupes sont faites au microtome $(10 \mu \mathrm{m})$, colorées avec du bleu alcian $8 \mathrm{GX}$ (Labosi) à 1 p. 100 et de la safranine $O$ (Labosi) à 1 p. 100 puis montées dans du baume du Canada. Les lames sont observées au microscope optique.

\section{RÉSULTATS}

\section{A. Choix d'un génotype témoin chez Regen $\mathbf{S}$}

Des différences très marquées ont été observées entre les 12 génotypes de Regen $\mathrm{S}$ testés. Elles portent sur la quantité de cals et sur l'aptitude à la régénération (tabl. 2).

Pour 3 génotypes (RS.11, RS.2 et RS.10), aucun signe de morphogenèse n'a jamais été observé, même après plusieurs mois de culture et de repiquages. Ces résultats ont toujours été confirmés lors d'expériences ultérieures.

TABLEAU 2

Aptitude à la régénération à partir de cals des génotypes Regen $S$. Regeneration ability from callus of Regen $S$ genotypes.

\begin{tabular}{|c|c|c|c|}
\hline Cienotype & $\begin{array}{l}\text { Callogenèse } \\
\text { (I) }\end{array}$ & $\begin{array}{c}\text { Indice } \\
\text { de croissance } \\
\text { des cals } \\
\text { (1) }\end{array}$ & $\begin{array}{c}\text { Morphogenèse } \\
\left({ }^{2}\right)\end{array}$ \\
\hline RS-1 & 90 & $+++t$ & 62 \\
\hline RS-3 & 87 & ++++ & 74 \\
\hline $\mathrm{RS}-6$ & 80 & $+t+t$ & 71 \\
\hline RS-5 & 58 & +++ & 31 \\
\hline RS-4 & 49 & $+t+$ & 33 \\
\hline RS-8 & 46 & ++ & 57 \\
\hline RS-11 & 40 & $+t$ & 0 \\
\hline RS-7 & 21 & ++ & 28 \\
\hline RS-2 & 19 & + & 0 \\
\hline RS -12 & 19 & + & 16 \\
\hline RS-9 & 13 & + & 23 \\
\hline RS-11) & 12 & ++ & 0 \\
\hline
\end{tabular}

N.B. : Effectif entre 100 et 120 explants par génotype. 100-120 explants for each genotype.

(1) Notations à $28 \mathrm{j}$ sur le milieu Bll.

Scored on BII medium after 28 days.

- Callogenèse : $\%$ d'explants donnant des cals.

- Callu formation : \% of explants forming callus.

- Indice de croissance des cals : $\quad+$ : de 0 à $2.5 \mathrm{~mm}$

- Growh index $++:$ de 2,5 à $5 \mathrm{~mm}$

+++ ; de 5 à $10 \mathrm{~mm}$

$+++t$ : plus de $10 \mathrm{~mm}$

(-) Notations après $28 \mathrm{j}$ sur le milieu BOI2Y

Morphogenèse : $\%$ des cals donnant des embryons ou des bourgeons.

Scored on BOI2Y medium after 28 days.

Morphogenesis : \% of callus forming embryos or buds.
Environ 70 p. 100 des cals issus des génotypes RS.1, RS. 3 et RS. 6 sont morphogènes et ont une vitesse de croissance élevée.

La taille des cals n'est pas liée à l'aptitude à régénérer : des cals de taille moyenne (RS.11, RS.10) ne produisent aucune néoformation alors que des cals de petite taille (RS.9, RS.12) peuvent donner des taux de différenciation de l'ordre de 20 p. 100.

La plante RS.3 a été choisie comme témoin dans la suite des expériences pour sa bonne aptitude à la morphogenèse.

\section{B. Comparaison de divers génotypes : aptitudes à l'embryogenèse somatique et à l'organogenèse}

Les résultats concernant l'aptitude à la régénération des 19 génotypes mis en comparaison sont donnés dans le tableau 3.

TABLEAU 3

Comportement en culture de tissus des différents génotypes. Callus production and regeneration efficiency of different genotypes.

\begin{tabular}{|c|c|c|c|c|}
\hline Génotype & $\begin{array}{l}\text { \%0 d'explants } \\
\text { donnant } \\
\text { des cals }\end{array}$ & $\begin{array}{c}\text { Indice } \\
\text { de croissance } \\
\text { des cals } \\
\text { (a) }\end{array}$ & $\begin{array}{l}\text { Régén } \\
\text { (a) }\end{array}$ & $\begin{array}{l}\text { ation } \\
\text { (c) }\end{array}$ \\
\hline RS-3 & 84 & $t+t$ & $E, P$ & 70 \\
\hline VN-1 & 56 & ++ & B & 0 \\
\hline VN-3 & 62 & ++ & $\mathrm{B}$ & 0 \\
\hline Tab 131 & 62 & ++ & & 0 \\
\hline Tab 71 & 38 & + & $\mathrm{C}$ & 0 \\
\hline $11 \times 84 b$ & 67 & ++ & $\mathrm{B}$ & 0 \\
\hline $11 \times 83$ & 65 & ++ & $\mathrm{B}$ & 0 \\
\hline Q $11 \times 104$ & 54 & ++ & B & 0 \\
\hline $8 \times 9$ & 39 & + & $\mathrm{C}$ & 0 \\
\hline $124-4$ & 31 & + & C & 0 \\
\hline $124-9$ & 48 & + & $\mathrm{C}$ & 0 \\
\hline $128-1$ & 53 & + & C & 0 \\
\hline $125-1$ & 91 & $++t$ & $E, P$ & 86 \\
\hline $125-2$ & 85 & +++ & $E, P$ & 72 \\
\hline D 12 & 13 & 0 & C & 0 \\
\hline D $5 \times$ D 1560 & 30 & + & $\mathrm{B}, \mathrm{P}$ & 8 \\
\hline D $15 \times$ D 5326 & 18 & 0 & $\mathrm{C}$ & 0 \\
\hline D $17 \times$ D 1514 & 15 & 0 & C & 0 \\
\hline $318-2$ & 43 & ++ & $\mathrm{B}, \mathrm{P}$ & 27 \\
\hline
\end{tabular}

N.B. : Nombre d'explants $=400$. 400 explants for each genotype.

(a) Indice de croissance des cals : $\quad+$ : de 2 à $5 \mathrm{~mm}$ Callus growth index $\quad++$ : de 5 à $10 \mathrm{~mm}$ +++ : plus de $10 \mathrm{~mm}$

(b) $\mathrm{E}$ : embryons, $\mathrm{P}$ : plantes ; $\mathrm{B}$ : bourgeons, $\mathrm{C}$ : cals. (c) $\%$ de cals produisant des plantes après 2 mois sur le milieu $\mathrm{BOI} 2 \mathrm{Y}=$ potentiel de régénération.

$\%$ of callus-producing plants after 2 months on BOI2Y medium $=$ regeneration potential.

Les différences entre les génotypes portent sur la callogenèse et sur la régénération. Tous les cals sont compacts sauf ceux issus des génotypes 125.1, 125.2, RS.3 et 318.2 dont les cals sont friables et à croissance rapide. Ce type de cals favorise d'ailleurs, chez ces 4 génotypes tétraploïdes, la régénération de plantes. Chez les génotypes diploïdes testés, la callogenèse est 


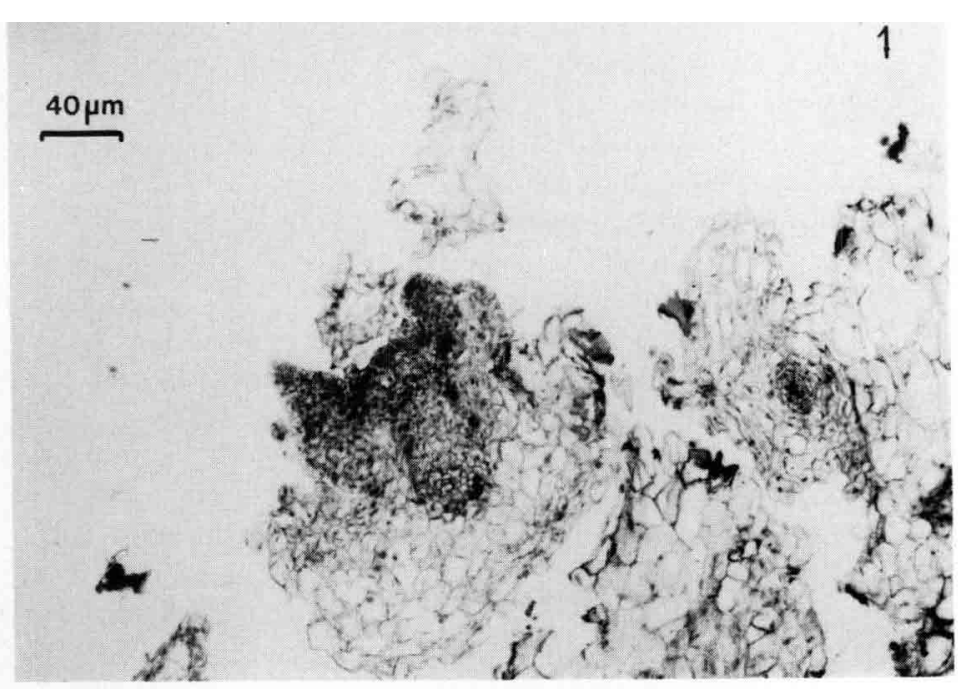

Figure 1

Coupe histologique d'un cal organogène avec méristème caulinaire. Section of an organogenic callus with shoot primordia.
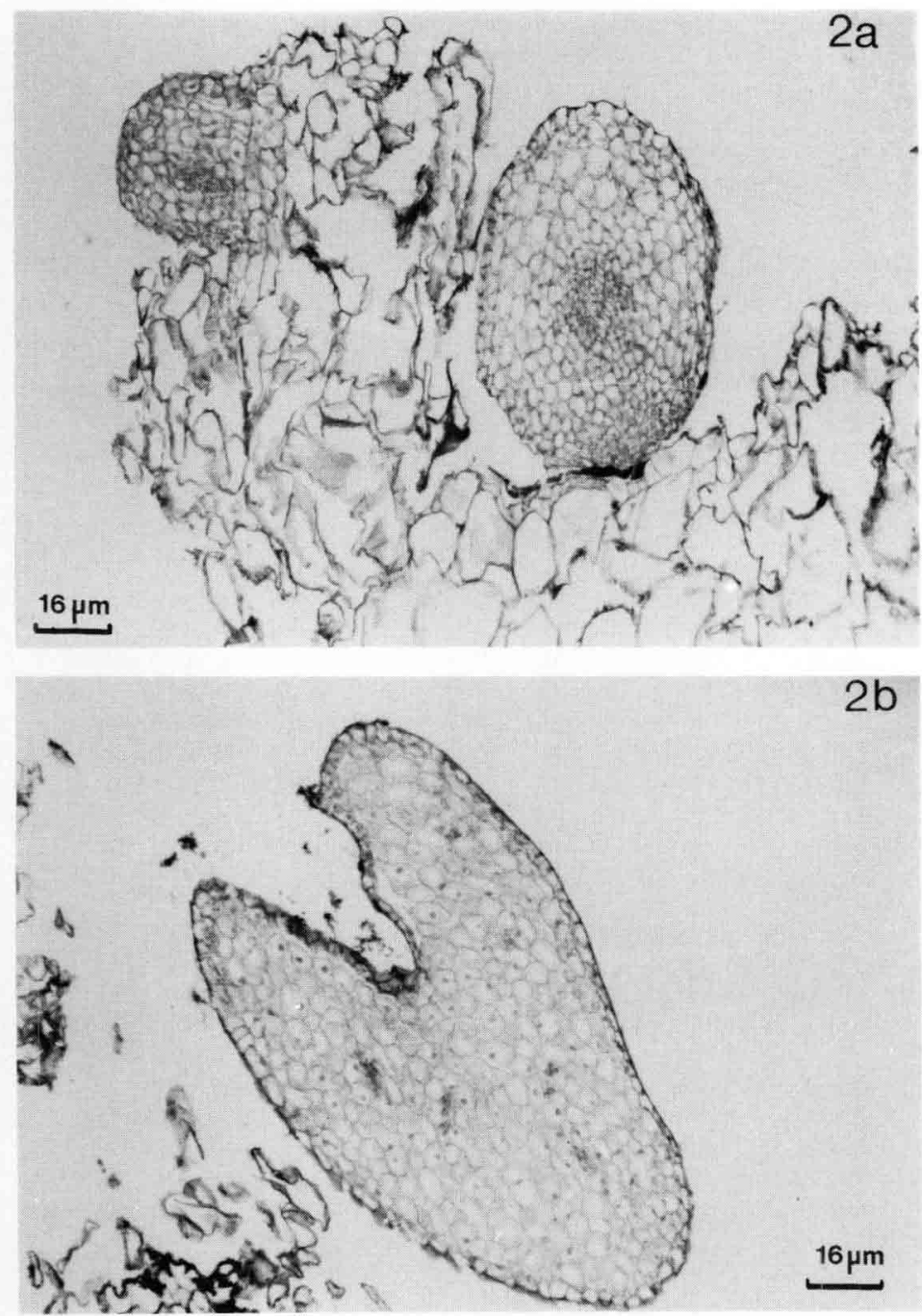

Figure 2

Coupes histologiques de cals embryogènes:

a. embryons globulaires,

b. embryon au stade cour.

Sections of embryos from embryogenic calli

a. globular embryos,

b. heart-shaped embryo. très réduite. Il n'a été possible de régénérer des plantes qu'à partir des cals issus de la plante D $5 \times \mathrm{D} 1560$.

$\mathrm{La}$ régénération de plantes peut se faire selon 2 voies exclusives dans les conditions testées : l'organogenèse ou l'embryogenèse somatique. Lors du processus organogénétique - observé sur les génotypes 318.2 et D $5 \times$ D 1560 - des massifs cellulaires d'un vert intense apparaissent à la surface des cals. Ils marquent un début d'organisation des cellules du cal en méristèmes (fig. 1). L'apparition séquentielle de bourgeons puis de tiges est ensuite observée : les premières feuilles sont trifoliolées, les racines et les tiges sont séparées par une masse de cal. 246 plantes ont ainsi été obtenues à partir du génotype 318.2 et 28 plantes à partir du génotype D $5 \times \mathrm{D} 1560$.

Les cals issus de RS.3, 125.1 et 125.2 ont permis l'obtention de plantes par embryogenèse somatique. Des excroissances blanchâtres et globulaires apparaissent à la surface des cals. Elles deviennent, par la suite, denses et très vertes puis se détachent très facilement du cal : il s'agit d'embryons avec un pôle racinaire et un pôle caulinaire.

Les coupes histologiques de ces embryons somatiques montrent qu'ils ont une structure semblable à celle des embryons zygotiques (fig. 2a et $2 \mathrm{~b}$ ). Plusieurs centaines d'embryons peuvent ainsi être obtenues sur un seul cal. Parmi les plantes testées, le génotype 125.1 surpasse tous les autres pour l'aptitude à la régénération en présentant un taux de développement d'embryons somatiques nettement plus élevé que RS.3.

La séquence de milieux UM-UMo, testée après les 2 premières expériences, a permis d'améliorer la qualité et la quantité des cals et surtout le rendement en embryons somatiques. De plus, sur cette séquence, les cals produisent un plus grand nombre d'embryons en un temps plus court : environ 5 semaines alors qu'il faut plus de 2 mois lorsque l'on utilise la séquence BII-BOI2Y.

Ces embryons sont mieux conformés que ceux obtenus sur la séquence BII-BOI2Y : les embryons à plusieurs cotylédons ou présentant des excroissances nombreuses sont rares.

A partir de génotypes très performants, les embryons peuvent apparaître directement sur le milieu UM sans repiquage sur UMo.

En comparant sur cette séquence de milieux plusieurs individus du cultivar "Europe " pour leur aptitude à l'embryogenèse somatique, une plante $E 1$, a été sélectionnée. La comparaison des potentiels embryogènes des génotypes RS.3. 125.1 et E1 montre que E1 est meilleure que RS.3 et très proche de 125.1 (tabl. 4). Le nombre d'embryons par cal est variable chez les 3 génotypes. Certains cals issus de RS.3 ne produisent pas d'embryons. Les écarts types sont assez importants, les cultures de E1 seraient plus homogènes mais le nombre moyen d'embryons reste plus élevé chez 125.1.

\section{DISCUSSION ET CONCLUSION}

Les présents résultats sur la formation des cals et l'embryogenèse somatique sont comparables à ceux 
TABLEAU 4

Comparaison des potentiels embryogènes de trois génotypes de luzerne.

Comparison of the embryogenic potential of 3 alfalfa genotypes.

\begin{tabular}{|c|c|c|c|c|c|}
\hline $\begin{array}{l}\text { Géno- } \\
\text { type }\end{array}$ & $\begin{array}{l}\text { Nombre } \\
\text { de cals } \\
\text { observés }\end{array}$ & $\begin{array}{l}\text { Nombre d' } \\
\text { par } \\
\text { Minimum }\end{array}$ & $\begin{array}{l}\text { 'embryons } \\
\text { cal } \\
\text { Maximum }\end{array}$ & $\begin{array}{c}\text { Nombre } \\
\text { total } \\
\text { d'embryons }\end{array}$ & $\begin{array}{c}\text { Nombre } \\
\text { moyen } \\
\text { d'embryons } \\
\text { par cal } \\
\pm \text { écart type }\end{array}$ \\
\hline RS 3 & 50 & 0 & 47 & 1066 & $21 \pm 15$ \\
\hline 125.1 & 49 & 3 & 118 & 3517 & $72 \pm 30$ \\
\hline E1 & 49 & 7 & 93 & 2802 & $57 \pm 21$ \\
\hline
\end{tabular}

N.B. : Séquence de milieux UM-UMo.

Première observation 15 jours après repiquage sur UMo puis régulièrement pendant 2 mois.

Sequence of media UM-UMo

First observation after 15 days on UMo, then regularly for 2 months.

publiés en culture de tissus de luzerne (BINGHAM et al., 1975 ; LUPOTTO, 1983 ; MITTEN et al., 1984 ; BROWN \& ATANASSOV, 1985) : la structure des cals et la fréquence de formation des embryons sont fortement influencées par le génotype de la plante. Les plantes étudiées ici sont d'origines diverses diploïdes et tétraploïdes de $M$. sativa et de $M$. falcata. Seuls 6 génotypes sur 19 ont permis la régénération de plantes à partir des cals. La quantité de cals n'a pas d'incidence sur la capacité à régénérer alors que la qualité des cals a une grande importance : les cals friables à croissance rapide sont les plus aptes à régénérer.

Des taux élevés d'embryogenèse somatique ont été obtenus sur les génotypes 125.1 d'une part et sur E1, issu du cultivar le plus cultivé en France (《Europe»), d'autre part.

Comme l'ont suggéré BingHAM et al. (1975) et BROWN \& ATANASSOV (1985), il semble possible de régénérer des plantes à partir de n'importe quelle population, sans sélection préalable, à condition de tester un nombre suffisant de plantes. Ainsi, Brown \& ATANASSOV (1985), à la suite d'essais extensifs sur des Medicago de toutes origines, observent 34 p. 100 de plantes aptes à produire des cals qui régénèrent. Ils en concluent une supériorité en culture in vitro de plantes d'une même origine géographique himalayenne (Ladak-Tibet) et des types à racines fasciculées comme M. falcata. Leur meilleur cultivar, « Rangelander », est fortement introgressé de $M$. falcata et ses fleurs sont bigarrées.

Le génotype 125.1, détecté ici comme le plus performant, est une $M$. sativa pure à fleurs violettes, tiges dressées et racines pivotantes. Toutefois, la population 125 porte le nom de "Mestnaya »; elle est, d'après SINSKAYA (1961), d'origine asiatique, et donc peut-être proche de l'origine Ladak-Tibet. La popula- tion "Europe " n'est constituée que de $M$. sativa de type flamand, donc légèrement introgressée de $M$. falcata, tout comme la population Regen S, qui elle, a été sélectionnée par BiNGHAM et al. (1975) pour l'aptitude à la régénération en culture in vitro.

Les résultats obtenus sur le seul génotype de $M$. falcata étudié ici (318.2) semblent montrer, qu'en effet, $M$. falcata est une espèce qui généralement se cultive bien in vitro. Toutefois, il faut souligner que ce génotype est tétraploïde et que, lors d'expériences préliminaires, aucun signe de régénération n'a été observé sur les cals obtenus à partir de 2 génotypes de $M$. falcata diplö̈des (M. falcata ssp. quasifalcata et $M$. falcata ssp. romanica).

L'efficacité de l'embryogenèse somatique est beaucoup plus grande que celle de l'organogenèse. On peut, en effet, obtenir un nombre très élevé d'embryons et de plantes, en un temps assez court ( 2 mois), issus d'un même génotype et ayant un âge physiologique proche de celui de plantules issues de graines. Le bouturage est une procédure assez aisée chez la luzerne mais ne fournit pas de matériel équivalent à de jeunes plantules.

Les études génétiques ou physiologiques des interactions entre un génotype hôte et un certain nombre de souches de pathogènes ou de Rhizobium peuvent, par exemple, être facilitées car les essais courants nécessitent l'inoculation et l'observation d'un grand nombre de plantules.

Cette technique permet également la multiplication rapide d'une plante intéressante, la conformité par rapport au clone initial restant toutefois à éprouver. La luzerne a d'ailleurs servi de plante modèle pour les études d'enrobages d'embryons somatiques en vue de la production de graines artificielles (K. WALKER, Y. DEMARLY, communications personnelles).

Cette étude a donc permis de trouver des génotypes ayant un très fort potentiel embryogène et de préciser les conditions d'obtention rapide d'embryons et de plantes. Ces génotypes sont actuellement utilisés dans des expériences d'hybridations somatiques avec d'autres espèces éloignées du genre Medicago et pour des expériences de transformation par Agrobacterium rhizogenes et Agrobacterium tumefaciens.

Reçu le 18 mars 1987. Accepté le 28 octobre 1987.

\section{REMERCIEMENTS}

Nous exprimons nos remerciements à D. BERGEon et H. FravÇoss pour leur aide technique. Ce travail a été réalisé dans le cadre de l'A.D.A.R. ( $\left.{ }^{*}\right)$ avec le soutien financier de l'A.C.V.F. $\left({ }^{* *}\right)$ et du Ministère de l'Agriculture.

(*) A.D.A.R. : Association pour le Développement des Applications de la Recherche en amélioration des plantes.

(**) A.C.V.F. : Association des Créateurs de Variétés Fourragères. 


\section{RÉFÉRENCES BIBLIOGRAPHIQUES}

Bingham E. T.. Hurley L. V., Kaatz D. M., Saunders J. W., 1975 Breeding alfalfa which regenerates from callus tissue in culture. Crop. Sci., 12, 719-721.

Brown D. C. W., Atanassov A., 1985. Role of genetic background in somatic embryogenesis in Medicago. Plant Cell Tissue Organ Culture, 4, 111-122.

Clement W. M., 1964. Stability of chromosome numbers in tissue cultures of alfalfa Medicago sativa. Amer. J. Bot., 51, 570.

Dos Santos A. V. P., Outka D. E., Cocking E. C., Davey M. R., 1980. Organogenesis and somatic embryogenesis in tissues derived from leaf protoplasts and leaf explants of Medicago sativa. $Z$. Pflanzenphysiol., 99, 261-270.

Kao K. N., Michayluk M. R., 1980. Plant regeneration from mesophyll protoplasts of alfalfa. Z. Pflanzenphysiol., 96, 135-141. Lupotto E., 1983. Propagation of an embryogenic culture of Medicago sativa L. Z. Pflanzenphysiol., 111, 95-104.

Mc Coy T. J., Bingham E. T., 1977. Regeneration of diploid alfalfa plants trom cells grown in suspension culture. Plant Sci. Lett., 10 , $59-66$.

Mitten D. H., Sato S. J., Skokut T. A., 1984. In vitro regenerative potential of alfalfa germplasm sources. Crop Sci., 24, 943-945.

Novak F. J., Konecna D., 1982. Somatic embryogenesis in callus and cell suspension cultures of alfalfa (Medicago sativa L.). $Z$. Pflanzenphysiol., 105, 279-284.

Saunders J. W., Bingham E. T., 1972. Production of alfalfa plants from callus tissue. Crop Sci., 12, 804-808.

Sinskaya E. N., 1961. Medicago. In « Flora of cultivated plants of the USSR ». Tome XIII : Perennial Leguminous Plants, 22-249, 326-343.

Teoule E., 1983. Hybridation somatique entre Medicago sativa L. et Medicago falcata. C. R. Acad. Sci. Paris, Sér. III, 297, 13-16.

Uchimiya H., Murashige T., 1974. Evaluation of parameters in the isolation of viable protoplasts from cultured tobacco cells. Plant Physiol., 54, 936-944. 